\title{
At what flow rate does the aortic valve gradient become severely elevated? Implications for guideline recommendations on aortic valve area cutoffs
}

\author{
Ayesha Salahuddin ${ }^{1}$, Wilbert S. Aronow ${ }^{2}$, Daniel M. Spevack ${ }^{1,2}$
}

${ }^{1}$ Department of Medicine, Cardiology Division, Montefiore Medical Center and Albert Einstein College of Medicine, Bronx, New York, USA

2Department of Cardiology, Westchester Medical Center and the Department

of Medicine, New York Medical College, Valhalla, New York, USA

Submitted: 3 December 2019; Accepted: 12 March 2020

Online publication: 21 March 2021

Arch Med Sci

DOI: https://doi.org/10.5114/aoms/118938

Copyright $\odot 2021$ Termedia \& Banach

\begin{abstract}
Introduction: Aortic stenosis (AS) is considered severe when the aortic valve area (AVA) is $<1.0 \mathrm{~cm}^{2}$ and the mean aortic valve gradient (mAVG) exceeds $40 \mathrm{~mm} \mathrm{Hg}$. Since many patients with AVA $<1.0 \mathrm{~cm}^{2}$ do not manifest an mAVG $>40 \mathrm{~mm} \mathrm{Hg}$, we sought to determine the AVA at which mAVG tends to exceed $40 \mathrm{~mm} \mathrm{Hg}$ in a sample of subjects with varied transvalvular flow rates. Material and methods: Our echocardiography database was queried for subjects with native valve AS. We selected 200 subjects with an AVA $<1.0 \mathrm{~cm}^{2}$. The sample was selected to include subjects with varied mean systolic flow (MSF) rates. Linear regression was performed to determine the relationship between MSF and MAVG. Since this relationship varied by AVA, the regression was stratified by AVA (critical $<0.6 \mathrm{~cm}^{2}$, severe $0.6-0.79 \mathrm{~cm}^{2}$, moderately severe $0.8-0.99 \mathrm{~cm}^{2}$ ).

Results: The study sample was $79 \pm 12$ years old and was $60 \%$ female. The MSF rate at which mAVG tended to exceed $40 \mathrm{~mm} \mathrm{Hg}$ was $120 \mathrm{ml} / \mathrm{s}$ for critical AVA, $183 \mathrm{ml} / \mathrm{s}$ for severe AVA and $257 \mathrm{ml} / \mathrm{s}$ for moderately severe AVA. Those with moderately severe AVA rarely (8\%) had an mAVG $>40 \mathrm{~mm} \mathrm{Hg}$ at a wide range of MSF. In contrast, those with severe AVA typically (75\%) had mAVG > $40 \mathrm{~mm} \mathrm{Hg}$ when MSF was normal (> $200 \mathrm{ml} / \mathrm{s}$ ). Those with critical AVA frequently (44\%) had mAVG $>40 \mathrm{~mm} \mathrm{Hg}$, even when MSF was reduced. Conclusions: Subjects with AVA of 0.8 and $0.9 \mathrm{~cm}^{2}$ rarely had mAVG $>40 \mathrm{~mm} \mathrm{Hg}$, even when the transvalvular flow rate was normal. Using current guidelines, it is not clear if such cases should be classified as severe.
\end{abstract}

Key words: low-flow, low-gradient aortic stenosis, mean systolic flow, ejection time.

\section{Introduction}

Current guideline recommendations state that aortic stenosis is severe when the aortic valve area (AVA) is less than $1.0 \mathrm{~cm}^{2}$ and the mean aortic valve gradient (mAVG) exceeds $40 \mathrm{~mm} \mathrm{Hg}$ [1, 2]. However, a great many patients who have an AVA less than $1.0 \mathrm{~cm}^{2}$ do not have an mAVG greater than $40 \mathrm{~mm} \mathrm{Hg}$ [3]. Such patients are often labeled as having low-flow, low-gradient aortic stenosis, since the presumed mechanism for having an mAVG less than $40 \mathrm{~mm} \mathrm{Hg}$ is that the flow rate across

\author{
Corresponding author: \\ Wilbert S. Aronow, MD, \\ FACC, FAHA \\ Department of Cardiology, \\ Westchester Medical Center \\ New York Medical College \\ Macy Pavilion, Room 141, \\ Valhalla, NY 10595, USA \\ Phone: (914)-493-5311 \\ Fax: (914)-235-6274 \\ E-mail: wsaronow@aol.com
}


the aortic valve is reduced due to abnormal left ventricular function or reduced left ventricular chamber volume $[4,5]$. Calculation of the transvalvular flow rate obviates the need for such assumptions. The mean systolic flow (MSF) rate may be calculated using echocardiography by dividing the measured stroke volume by the systolic ejection period duration $[6,7]$. In the case of low-flow, low-gradient aortic stenosis with reduced MSF, provocative testing may be used to test whether the stenosis remains truly severe when the transvalvular flow rate is improved with an inotrope such as dobutamine [8]. However, when the mAVG is less than $40 \mathrm{~mm} \mathrm{Hg}$ in the presence of preserved systolic flow, it is uncertain whether the stenosis should be classified as hemodynamically severe. Accordingly, we sought to determine the AVA at which mAVG tends to exceed $40 \mathrm{~mm} \mathrm{Hg}$ in a sample of subjects with varied transvalvular flow rates.

\section{Material and methods}

Our institutional echocardiography database was queried using Apollo (a cardiovascular clinical data repository) for all subjects with native valve aortic stenosis who had echocardiograms performed from 2010 to 2012. The clinical outcomes from this sample of 4,546 patients were previously published [9]. From that sample, we selected 200 consecutive subjects with an AVA less than $1.0 \mathrm{~cm}^{2}$ based on the echocardiography report. Patients with severe mitral valve disease and severe aortic regurgitation were excluded. Subjects were assigned to 3 groups: (1) high gradient aortic stenosis (HGAS): mAVG > $40 \mathrm{~mm}$ $\mathrm{Hg}(n=50)$; (2) low-gradient aortic stenosis with normal left ventricular ejection fraction (LGNEF): mAVG $<40 \mathrm{~mm} \mathrm{Hg}$, and LVEF $\geq 50 \% n=100$ ), and (3) low-gradient aortic stenosis with low left ventricular ejection fraction (LGLEF): mAVG $<40 \mathrm{~mm} \mathrm{Hg}$ and left ventricular ejection fraction $<50 \%(n=50)$.

Because LGNEF was a group of particular interest, we preferentially allocated subjects to this group. We suspected a priori that many of these subjects would have hemodynamics suggestive of moderate aortic stenosis. Two-dimensional and Doppler echocardiographic studies had been performed on commercially available ultrasound equipment (Philips iE33, Andover, Massachusetts). A single reader re-measured stroke volume, mAVG and systolic ejection time. The AVA was determined by the continuity equation. Peak and mean transvalvular pressure gradients were derived from the modified Bernoulli equation, measured in the apical 5-chamber view. The biplane Simpson's method and the Doppler VTI method $\left(\pi \mathrm{r}_{\text {LVOT }}^{2} \times \mathrm{VTI}_{\text {LVOT }}\right)$ were both used to calculate the stroke volume. Systolic ejection period (SEP) was measured from continuous-wave Doppler of the

Table I. Patient characteristics and comorbidities

\begin{tabular}{|c|c|c|c|c|c|}
\hline \multirow[t]{2}{*}{ Parameter } & \multirow{2}{*}{$\begin{array}{l}\text { High gradient } \\
\text { AS }(n=52) \\
(1)\end{array}$} & \multirow{2}{*}{$\begin{array}{c}\text { LGNEF } \\
(n=109) \\
(2)\end{array}$} & \multirow{2}{*}{$\begin{array}{l}\text { LGLEF } \\
(n=39) \\
(3)\end{array}$} & \multicolumn{2}{|c|}{$P$-values comparing columns } \\
\hline & & & & 1 vs. 2 & 1 vs. 3 \\
\hline \multicolumn{6}{|l|}{ Characteristics: } \\
\hline Age [years] & $78 \pm 13$ & $82 \pm 11$ & $74 \pm 12$ & 0.06 & 0.20 \\
\hline Male (\%) & 36 & 34 & 56 & 0.83 & 0.06 \\
\hline Height [inches] & $64 \pm 5$ & $62 \pm 4$ & $65 \pm 4$ & 0.06 & 0.13 \\
\hline Weight [pounds] & $163 \pm 42$ & $146 \pm 34$ & $154 \pm 3$ & 0.007 & 0.29 \\
\hline BMI $\left[\mathrm{kg} / \mathrm{m}^{2}\right]$ & $28 \pm 6$ & $27 \pm 7$ & $26 \pm 5$ & 0.18 & 0.04 \\
\hline $\mathrm{SBP}[\mathrm{mm} \mathrm{Hg}]$ & $128 \pm 20$ & $135 \pm 21$ & $120 \pm 21$ & 0.08 & 0.04 \\
\hline $\mathrm{DBP}[\mathrm{mm} \mathrm{Hg}]$ & $65 \pm 11$ & $65 \pm 14$ & $68 \pm 10$ & 0.73 & 0.06 \\
\hline \multicolumn{6}{|l|}{ Comorbidities (\%): } \\
\hline Hypertension & 69 & 74 & 69 & 0.50 & 0.99 \\
\hline Hyperlipidemia & 32 & 37 & 36 & 0.62 & 0.75 \\
\hline Coronary artery disease & 36 & 40 & 56 & 0.64 & 0.06 \\
\hline Diabetes & 21 & 34 & 36 & 0.10 & 0.12 \\
\hline CVA & 4 & 13 & 13 & 0.06 & 0.11 \\
\hline COPD & 6 & 7 & 10 & 0.72 & 0.43 \\
\hline PVD & 2 & 4 & 13 & 0.55 & 0.04 \\
\hline Chronic kidney disease & 15 & 20 & 28 & 0.46 & 0.14 \\
\hline
\end{tabular}

$n$ - number of subjects, LGNEF - low-gradient normal ejection fraction, LGLEF-low-gradient low ejection fraction, BMI - body mass index, $S B P$ - systolic blood pressure, DBP - diastolic blood pressure, CVA - cerebrovascular accident, COPD - chronic obstructive pulmonary disease, $P V D$ - peripheral vascular disease. 
left ventricular outflow tract (LVOT). Mean systolic flow (MSF) was calculated using the formula: $\pi r^{2}{ }_{\text {LVOT }}$ $\times \mathrm{VTI}_{\text {LVOT }} / \mathrm{SEP}$. If mAVG or LVEF differed between the study reader and the values reported on the echo reports, the subjects were re-categorized into the appropriate group based on the study reader's findings (Table I).

Medical records were reviewed for clinical characteristics and co-morbidities. Montefiore Medical Center's Institutional Review Board approved the study.

\section{Statistical analysis}

Statistical analysis was done using Stata software, version 11 (College Station, TX). Normally distributed data were presented as the mean \pm standard deviation (SD). Comparison of means was performed using the two-sample $t$-test. Comparison of categorical data was performed using the $\chi^{2}$ test. $P$-values were considered significant if $<0.05$. Linear regression was performed to determine the relationship between MSF and mAVG. Since this relationship varied by AVA, the regression was stratified by AVA into the following 3 groups: (1) moderately severe AVA: AVA 0.80$0.99 \mathrm{~cm}^{2}$; (2) severe AVA: AVA 0.60-0.79 $\mathrm{cm}^{2}$; and (3) critical AVA: AVA $<0.60 \mathrm{~cm}^{2}$.

It should be noted that the relationship between mAVG and MSF is known not to be linear. As shown in the Gorlin equation, mAVG is related to the square of the MSF for a given AVA. AVA = MSF $\div(44.3 \times \sqrt{ } \mathrm{mAVG})$, where 44.3 is the empirically derived discharge coefficient. Stated otherwise, $\mathrm{mAVG}=\mathrm{MSF}^{2} \div(44.3 \times \mathrm{AVA})^{2}$. While current guidelines define a low-flow state in terms of the stroke volume index, we chose to use MSF be- cause it is independent of body size and because its use in the Gorlin equation demonstrates that this parameter is the key variable linking aortic valve gradient to aortic valve area.

We used linear regression because this relationship is known to be nearly linear for the range of MSF found in physiologic states and also because our intent was to simply estimate the MSF at which $\mathrm{mAVG}$ tended to be greater than $40 \mathrm{~mm} \mathrm{Hg}$ for a given AVA.

Inter-observer variability was measured by comparing AVA measurements performed by the study investigator to those reported on the clinical reports. Variability was assessed using Bland-Altman analysis to test for bias and agreement between readers.

\section{Results}

The clinical characteristic and co-morbidities of patients in each group are listed in Table I. Among the 200 subjects, 52 patients had high-gradient aortic stenosis, 109 patients had LGNEF aortic stenosis, and 39 patients had LGLEF aortic stenosis. Compared to the HGAS group, the LGNEF group had more females, was older and had a significantly lower body surface area. The incidence of peripheral vascular disease was higher in the LGLEF group.

Table II summarizes the echocardiographic parameters of the 3 groups. Compared to the HGAS group, the LGNEF group had a significantly lower peak gradient, lower end-diastolic volume, lower stroke volume, shorter ejection time and lower MSF. Compared with the HGAS group, the LFLEF group had a lower peak gradient, higher end-diastolic volume, lower stroke volume, larg-

Table II. Echocardiographic parameters

\begin{tabular}{|c|c|c|c|c|c|}
\hline \multirow[t]{2}{*}{ Parameter } & \multirow{2}{*}{$\begin{array}{l}\text { High gradient } \\
\text { AS }(n=52) \\
(1)\end{array}$} & \multirow{2}{*}{$\begin{array}{c}\text { LGNEF } \\
(n=109) \\
(2)\end{array}$} & \multirow{2}{*}{$\begin{array}{c}\text { LGLEF } \\
(n=39) \\
(3)\end{array}$} & \multicolumn{2}{|c|}{$P$-values comparing columns } \\
\hline & & & & 1 vs. 2 & 1 vs. 3 \\
\hline Ejection fraction (\%) & $62 \pm 11$ & $63 \pm 7$ & $32 \pm 9$ & 0.38 & $<0.001$ \\
\hline Aortic valve area $\left[\mathrm{cm}^{2}\right]$ & $0.63 \pm 0.15$ & $0.76 \pm 0.12$ & $0.72 \pm 0.16$ & $<0.001$ & 0.008 \\
\hline Peak gradient [mm Hg] & $88 \pm 18$ & $46 \pm 14$ & $40 \pm 15$ & $<0.001$ & $<0.001$ \\
\hline Mean gradient [mm Hg] & $56 \pm 12$ & $26 \pm 8$ & $23 \pm 9$ & $<0.001$ & $<0.001$ \\
\hline EDV [ml] & $90 \pm 28$ & $75 \pm 26$ & $131 \pm 46$ & $<0.001$ & $<0.001$ \\
\hline SV biplane [ml] & $58 \pm 17$ & $47 \pm 12$ & $44 \pm 12$ & $<0.001$ & $<0.001$ \\
\hline SV LVOT [ml] & $61 \pm 17$ & $50 \pm 12$ & $42 \pm 11$ & $<0.001$ & $<0.001$ \\
\hline $\mathrm{SVi}\left[\mathrm{ml} / \mathrm{m}^{2}\right]$ & $34 \pm 9$ & $30 \pm 7$ & $24 \pm 6$ & 0.003 & $<0.001$ \\
\hline Ejection time $[\mathrm{ms}]$ & $330 \pm 30$ & $310 \pm 40$ & $290 \pm 30$ & 0.030 & $<0.001$ \\
\hline MSF [ml/s] & $184 \pm 52$ & $159 \pm 37$ & $145 \pm 33$ & $<0.001$ & $<0.001$ \\
\hline LVOT diameter [cm] & $1.84 \pm 0.22$ & $1.78 \pm 0.21$ & $1.95 \pm 0.19$ & 0.39 & 0.03 \\
\hline Heart rate [beats/min] & $74 \pm 13$ & $73 \pm 13$ & $78 \pm 14$ & 0.86 & 0.14 \\
\hline
\end{tabular}

$n$ - number of subjects, AS - aortic stenosis, LGNEF - low-gradient normal ejection fraction, LGLEF-low-gradient low ejection fraction, EDV - end diastolic volume, SV biplane - stroke volume biplane Simpson's method, LVOT - left ventricular outflow tract, MSF - mean systolic flow. 


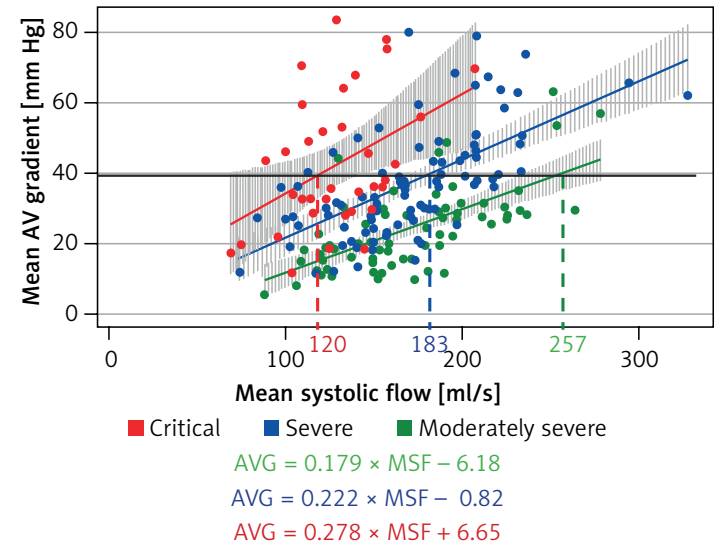

Figure 1. Linear regression between mean systolic flow (MSF) and mean aortic valve gradient (mAVG). Solid line indicates mAVG of $40 \mathrm{~mm} \mathrm{Hg}$. Interrupted lines indicate the MSF when critical (red), severe (blue) and moderately severe (green) AVA group cross the gradient of $40 \mathrm{~mm} \mathrm{Hg}$

er LVOT diameter, shorter ejection time, and lower MSF.

For reasons outlined earlier, linear regression was performed to determine the relationship between MSF and mAVG (Figure 1). Since this relationship varied by AVA, the 3 groups were further stratified by AVA into moderately severe AVA, severe AVA, and critical AVA. The echocardiographic parameters for each group are shown in Table III. When compared with the moderately severe AVA group, the severe AVA group had a higher peak gradient, higher mean gradient, and longer ejection time. The critical AVA group had a higher peak and mean gradient, lower stroke volume and stroke volume index, longer ejection time and lower mean systolic flow.
The MSF rate at which mAVG tended to be greater than $40 \mathrm{~mm} \mathrm{Hg}$ was $120 \mathrm{ml} / \mathrm{s}$ in those with critical AVA, $183 \mathrm{ml} / \mathrm{s}$ in those with severe AVA and $257 \mathrm{ml} / \mathrm{s}$ in those with moderately severe AVA (Figure 1). When the MSF exceeded $200 \mathrm{ml} / \mathrm{s}$, nearly all of those with critical or severe AVA had an mAVG at or near $40 \mathrm{~mm} \mathrm{Hg}$. For this reason, we chose this cutoff to represent preserved systolic flow rate.

The mAVG exceeded $40 \mathrm{~mm} \mathrm{Hg}$ in $46 \%, 33 \%$ and $8 \%$ in those with critical, severe and moderately severe AVA, respectively. In the subset with preserved MSF, the mAVG exceeded $40 \mathrm{~mm} \mathrm{Hg}$ in $100 \%, 75 \%$ and $21 \%$ in those with critical, severe and moderately severe AVA, respectively. All of the subjects in the severe and critical AVA group had an $\mathrm{mAVG} \geq 37 \mathrm{~mm} \mathrm{Hg}$ when MSF was preserved.

Clinical echocardiography reports tended to over-estimate AVA compared to the study reader (0.72 vs. $\left.0.66 \mathrm{~cm}^{2}, p<0.001\right)$. The standard deviation of the difference between the readers was $0.12 \mathrm{~cm}^{2}$. In a normal distribution, one standard deviation accounts for $68 \%$ of all values. Therefore, in most cases (i.e. $68 \%$ of the time), the study reader and the clinical reader agreed on the AVA within $0.12 \mathrm{~cm}^{2}$. The clinical reports tended to over-estimate the SV compared to the study reader ( 56.9 vs. $51.1 \mathrm{ml}, p<0.001)$. The standard deviation of the mean difference between the study reader and the clinical reports was $5.8 \mathrm{ml}$. The study reader over-estimated the SV using the LVOT Doppler method $\left(\pi \mathrm{r}^{2}{ }_{\text {LVOT }} \times \mathrm{VTI}_{\text {LVOT }}\right)$ compared to the SV measured using the biplane Simpson's method (51.1 vs. $49.2 \mathrm{ml}, p<0.001$ ). The standard deviation of the mean difference between the methods was $7.1 \mathrm{ml}$.

Table III. Echocardiographic parameters based on aortic valve area

\begin{tabular}{|c|c|c|c|c|c|}
\hline \multirow[t]{2}{*}{ Parameter } & \multirow{2}{*}{$\begin{array}{c}\text { Moderately severe AS } \\
\left(\text { AVA } 0.8-0.99 \mathrm{~cm}^{2}\right) \\
(n=75) \\
(1)\end{array}$} & \multirow{2}{*}{$\begin{array}{c}\text { Severe AS } \\
\left(\text { AVA } 0.60-0.79 \mathrm{~cm}^{2}\right) \\
(n=90) \\
(2)\end{array}$} & \multirow{2}{*}{$\begin{array}{c}\text { Critical AS } \\
\left(\text { AVA } \leq 0.59 \mathrm{~cm}^{2}\right) \\
(n=35) \\
(3)\end{array}$} & \multicolumn{2}{|c|}{$\begin{array}{l}P \text {-values comparing } \\
\text { columns }\end{array}$} \\
\hline & & & & 1 vs. 2 & 1 vs. 3 \\
\hline Ejection fraction (\%) & $57 \pm 13$ & $58 \pm 15$ & $52 \pm 15$ & 0.64 & 0.11 \\
\hline Aortic valve area $\left[\mathrm{cm}^{2}\right]$ & $0.86 \pm 0.04$ & $0.69 \pm 0.05$ & $0.46 \pm 0.08$ & $<0.001$ & $<0.001$ \\
\hline Peak gradient [mm Hg] & $42 \pm 18$ & $61 \pm 22$ & $71 \pm 30$ & $<0.001$ & $<0.001$ \\
\hline Mean gradient [mm Hg] & $24 \pm 11$ & $37 \pm 15$ & $43 \pm 19$ & $<0.001$ & $<0.001$ \\
\hline EDV [ml] & $85 \pm 33$ & $95 \pm 43$ & $86 \pm 30$ & 0.12 & 0.95 \\
\hline SV biplane [ml] & $48 \pm 13$ & $52 \pm 16$ & $42 \pm 12$ & 0.08 & 0.03 \\
\hline SV LVOT [ml] & $51 \pm 15$ & $54 \pm 14$ & $41 \pm 9$ & 0.22 & $<0.001$ \\
\hline SV index (LVOT) & $30 \pm 9$ & $31 \pm 8$ & $25 \pm 6$ & 0.67 & 0.002 \\
\hline Ejection time [ms] & $304 \pm 40$ & $319 \pm 30$ & $322 \pm 30$ & 0.02 & 0.05 \\
\hline MSF $[\mathrm{ml} / \mathrm{s}]$ & $169 \pm 41$ & $171 \pm 44$ & $129 \pm 29$ & 0.79 & $<0.001$ \\
\hline LVOT diameter [cm] & $1.8 \pm 0.21$ & $1.8 \pm 0.23$ & $1.74 \pm 0.18$ & 0.27 & 0.10 \\
\hline Heart rate [beats/min] & $73 \pm 12$ & $74 \pm 14$ & $75 \pm 13$ & 0.43 & .040 \\
\hline
\end{tabular}

$n$ - number of subjects, AVA - aortic valve area, EDV - end diastolic volume, SV biplane - stroke volume biplane Simpson's method, LVOT - left ventricular outflow tract, MSF - mean systolic flow. 


\section{Discussion}

Our main finding is that subjects with an AVA between 0.8 and $1.0 \mathrm{~cm}^{2}$ rarely have an mAVG $>40 \mathrm{~mm} \mathrm{Hg}$. This is true even when the MSF is preserved (i.e. $>200 \mathrm{ml} / \mathrm{s}$ ). This finding is in agreement with the Gorlin estimates for subjects with such hemodynamics. According the Gorlin equation, a subject with an AVA of $0.8 \mathrm{~cm}^{2}$, who has a normal MSF of $200 \mathrm{ml} / \mathrm{s}$, would have a calculated mAVG of $32 \mathrm{~mm} \mathrm{Hg}$. If the guidelines continue to advocate using AVA $<1.0 \mathrm{~cm}^{2}$ as a criterion for severe stenosis, it may therefore be reasonable to lower the cutoff value for mAVG to about $30-35 \mathrm{~mm} \mathrm{Hg}$ for AS to be considered severe.

We suggest that when the AVA $<1.0 \mathrm{~cm}^{2}$ and the mAVG $<40 \mathrm{~mm} \mathrm{Hg}$, it is important to calculate the MSF. Without this parameter it is difficult to know whether the patient truly has low flow causing the discrepancy between valve area and valve gradient. While the current guidelines have focused on stroke volume index instead of systolic flow rate, this parameter may be misleading [1, 2]. We found that $25 \%$ of subjects with preserved MSF had an SVI $<35 \mathrm{ml} / \mathrm{m}^{2}$. In a previous retrospective study analyzing stress echocardiography performed for low-flow, low-gradient AS, flow rate was found to be the only resting systolic parameter independently associated with severe AS [9].

In our study, we chose a cutoff value for mean systolic flow rate above $200 \mathrm{ml} / \mathrm{s}$ to be considered preserved. At this flow rate, nearly all of those with critical or severe AVA had an MAVG at or near $40 \mathrm{~mm} \mathrm{Hg}$. Other authors have also endorsed using this cutoff [9-12]. Voelker et al. conducted an in vitro study in a pulsatile aortic flow model in which they derived the mean systolic flow from electromagnetic flow curves [13]. They considered a systolic flow of $200 \mathrm{ml} / \mathrm{s}$ as physiologic, as it corresponded to a cardiac output of $5 \mathrm{l} / \mathrm{min}$ [13]. The authors of the multicenter Truly or Pseudo-Severe Aortic Stenosis (TOPAS) study considered a cutoff of $250 \mathrm{ml} / \mathrm{s}$ to be the normal transvalvular flow rate [6]. In our sample, however, only 6 of the included subjects had an MSF at or above this cutoff. Although our sample was selected to be enriched with low-flow aortic stenosis, it seems that the use of a cutoff of $250 \mathrm{ml} / \mathrm{s}$ is very high, since the vast majority of subjects with severe aortic stenosis do not reach this level of flow. Irrespective of what cutoff of MSF is ultimately determined to be the optimal, our feeling is that a patient should not be labeled as having low flow if their MSF exceeds the cutoff value.

This study does have limitations. The study design is retrospective, and it included a selected sample of subjects purposefully enriched with having low-gradient aortic stenosis. The sample also excluded those who had severe aortic or mitral regurgitation. These factors may limit the generalizability of our results. Another limitation is that measurement error may play a role in misclassification of patients with aortic stenosis. We measured important differences in inter-observer and intra-observer variability for AVA and SV. Therefore some of the subjects with low-gradient aortic stenosis may have had true AVA $>1.0 \mathrm{~cm}^{2}$.

In conclusion, subjects with AVA of 0.8 and $0.9 \mathrm{~cm}^{2}$ rarely had $\mathrm{mAVG}>40 \mathrm{~mm} \mathrm{Hg}$, even when the transvalvular flow rate was normal. Using current guidelines, it is not clear whether such cases should be classified as severe.

\section{Conflict of interest}

The authors declare no conflicts of interest.

\section{References}

1. Nishimura RA, Otto CM, Bonow RO, et al. 2014 AHA/ ACC guideline for the management of patients with valvular heart disease: a report of the American College of Cardiology/American Heart Association Task Force on Practice Guidelines. J Thorac Cardiovasc Surg 2014; 148: e1-e132.

2. Baumgartner H, Falk V, Bax JJ, et al. 2017 ESC/EACTS guidelines for the management of valvular heart disease. Eur Heart J 2017; 38: 2739-91.

3. Minners J, Aligeier M, Gohlke-Baerwolf C, et al. Inconsistencies of echocardiographic criteria for the grading of aortic valve stenosis. Eur Heart J 2008; 29: 1043-8.

4. Eleid MF, Sorajja P, Michelena HI, et al. Flow-gradient patterns in severe aortic stenosis with preserved ejection fraction: clinical characteristics and predictors of survival. Circulation 2013; 128: 1781-9.

5. Clavel MA, Magne J, Pibarot P. Low-gradient aortic stenosis. Eur Heart J 2016; 37: 2645-57.

6. Blais C, Burwash IG, Mundigier G, et al. Projected valve area at normal flow rate improves the assessment of stenosis severity in patients with low-flow, low-gradient aortic stenosis: the multicenter TOPAS (Truly or Pseudo-Severe Aortic Stenosis) study. Circulation 2006; 113: 711-21.

7. Hachicha Z, Dumesnil JG, Bogaty P, Pibarot P. Paradoxical low-flow, low-gradient severe aortic stenosis despite preserved ejection fraction is associated with higher afterload and reduced survival. Circulation 2007; 115: 2856-64.

8. Ploriska-Gosciniak E, Lipiec P, Lancellotti P, et al. Prognostic value of low-dose dobutamine stress echocardiography in patients with aortic stenosis and impaired left ventricular function. Arch Med Sci 2013; 9: 434-9.

9. Romero J, Chavez P, Goodman-Meza D, et al. Outcomes in patients with various forms of aortic stenosis including those with low-flow low-gradient normal and low ejection fraction. Am J Cardiol 2014; 114: 1069-74.

10. Chahal NS, Drakopoulou M, Gonzalez-Gonzalez AM, Manivarmane R, Khattar R, Senior R. Resting aortic valve area at normal transaortic flow rate reflects true valve area in suspected low-gradient severe aortic stenosis. JACC Cardiovasc Imaging 2015; 8: 1133-9.

11. Saeed S, Senior R, Chahai NS, et al. Lower transaortic flow rate Is associated with increased mortality in aortic valve stenosis. JACC Cardiovasc Imaging 2017; 10: 912-20. 
12. Vamvakidou A, Chahal N, Senior R. Lack of stroke volume determined flow reserve does not always preclude assessment of severity of aortic stenosis in low-flow low-gradient state during dobutamine echocardiography. JACC Cardiovasc Imaging 2017; 10: 491-3.

13. Voelker W, Reul H, Nienhaus G, et al. Comparison of valvular resistance, stroke work loss, and Gorlin valve area for quantification of aortic stenosis. An in vitro study in a pulsatile aortic flow model. Circulation 1995; 91: 1196-204. 